\title{
The Mesenchymal-Epithelial and Epithelial-Mesenchymal Cellular Plasticity of Liver Metastases with Digestive Origin
}

\author{
AMALIA RALUCA CEAUSU ${ }^{1}$, ALEXANDRU CIOLOFAN ${ }^{1,2}$, ANCA MARIA CIMPEAN ${ }^{1}$, \\ ADINA MAGHETI ${ }^{1}$, OVIDIU MEDERLE $^{1}$ and MARIUS RAICA ${ }^{1}$ \\ ${ }^{1}$ Department of Microscopic Morphology/Histology, Angiogenesis Research Center Timisoara, and \\ ${ }^{2}$ Department of Surgery, Victor Babes University of Medicine and Pharmacy, Timisoara, Romania
}

\begin{abstract}
Background: Few data are available regarding the epithelial to mesenchymal transition (EMT) /mesenchymal to epitheilal transition (MET) in the liver metastasis of digestive cancers. The aim of this study was to establish EMT/MET metastatic tumor cell plasticity according to the histological growth pattern of liver metastases. Materials and Methods: Biopsies from 25 patients with liver metastasis (desmoplastic, replacement and pushing type) were evaluated. Double immunostaining of E-cadherin/vimentin, keratin 8,18/vimentin and E-cadherin/ keratin 8,18 were performed. Results: The following cell types were noted: epithelial, mesenchymal, non-differentiated and differentiated hybrid mesenchymal/ epithelial and non-hybrid phenotype. All cases had mesenchymall epithelial phenotype cells. A significant correlation was found between the non-differentiated hybrid mesenchymall epithelial phenotype metastatic cells and histological growth pattern for gastric and colorectal cancer. Conclusion: A MET-targeting strategy, in conjunction with conventional chemotherapy, may be useful for the treatment of liver metastases.
\end{abstract}

The epithelial to mesenchymal transition (EMT) represents the conversion of epithelial cell phenotype to mesenchymal cell phenotype, which is characterized by elongated, spindleshaped cells. The mesenchymal to epithelial transition (MET) is the reverse phenomenon. There are three cell phenotypes described in the EMT/MET process: epithelial, mesenchymal and a hybrid epithelial/mesenchymal (partial or intermediate EMT) phenotype (1).

Correspondence to: Professor Anca Maria Cimpean, MD, Ph.D., Victor Babes University of Medicine and Pharmacy, Department of Microscopic Morphology/Histology, Angiogenesis Research Center Timisoara, Piata Eftimie Murgu no. 2, 300041, Timisoara, Timis, Romania. Tel: +40 256204476, e-mail: ancacimpean1972@yahoo.com

Key Words: Epithelial-mesenchymal transition, mesenchymalepithelial transition, liver metastases.
EMT was described in embryogenesis and organ development in physiological situations (2-4). In malignant lesions, EMT involves cytoskeletal disorders, loss of cellcell adhesion and apical-basal cell polarity. In colorectal carcinoma, EMT was found in the cells from the invasive front (5). In pancreatic adenocarcinoma, loss of E-cadherin expression was noted in well- and poorly differentiated ductal adenocarcinoma, and few or none of the undifferentiated carcinomas $(6,7)$. Fewer than $50 \%$ of gastric cancer cells express E-cadherin. It was shown that the diffuse-type gastric cancer is associated with the Ecadherin/N-cadherin switch, whereas the intestinal-type is related to up-regulation of transforming growth factor beta (TGF) $\beta /$ loss of E-cadherin (8).

Data from the literature show, using murine experimental models of metastasis, the presence of MET phenomenon in liver, lung, and brain metastases (9-11). Liver metastases from prostate cancer showed epithelial morphology in most cases (12). In liver metastases of colorectal adenocarcinoma, the increased expression of E-cadherin and decreased vimentin expression was noted $(13,14)$. More recently, in an experimental model, the involvement of sciellin as an inducer of MET through the liver metastasis process of colorectal cancer was shown (15). It increased Wnt signaling and favored MET through the sciellin- $\beta$-catenin-E-cadherin axis. Thus, in both mouse and human pancreatic ductal adenocarcinoma, metastatic cells appear to re-acquire an epithelial phenotype with increasing lesion size. Immunohistochemical analysis revealed a higher immunoexpression intensity of claudin 7 in primary tumors than in micro-metastases. Increased immunoexpression of fibroblast-specific protein 1 was found in micro-metastases compared to gross metastases and primary tumors (16).

The replacement growth pattern was suggested as being prevalent in liver metastases with pancreatic origin (17) and the pushing type for the gastric origin (18). The following types of growth were described for liver metastases of colorectal cancer: replacement, pushing, desmoplastic and mixed. The prognostic role of the histological growth pattern 
of liver metastasis was shown at diagnosis but also after portal vein embolization in colorectal cancer $(19,20)$.

The aim of this study was to establish metastatic tumor cell plasticity according to the histological growth pattern of liver metastases with colorectal, pancreatic and gastric origins.

\section{Materials and Method}

The present study included 25 patients who underwent surgery on between 2009-2016. Liver metastasis was of different origins: colorectal adenocarcinoma in 15 cases, pancreatic in seven cases and gastric adenocarcinoma in three cases. Signed consent was obtained from each patient included in this study.

All procedures were carried out according to the principles of the Declaration of Helsinki and were approved by the Institutional Review Board (no. 7339/22.04.2016). The patients included in the study underwent excisional tumorectomy of the liver metastasis. Metastatic fragments were fixed in $10 \%$ buffered formalin for $24 \mathrm{~h}$ and paraffin embedded. Morphological and immunohisto-chemical staining were performed.

Double immunostainings for keratin 18/vimentin and Ecadherin/vimentin were used. Heat-induced epitope retrieval with Bond Epitope Retrieval Solution 2 (ready-to-use, $\mathrm{pH}$ 9.0; Leica Biosystems, Newcastle Ltd, Newcastle upon Tyne, UK) for 20 minutes was the first step. Endogenous peroxidase blocking was achieved with $3 \%$ hydrogen peroxide for 5 minutes. The following primary antibodies were used: keratin 8,18 (monoclonal, clone 5 D3, ready to use), E-cadherin (monoclonal, clone 36B5, ready to use), vimentin (monoclonal, clone V9, ready to use). All antibodies used were from Leica Bond Biosystems. The Bond Polymer Refine Detection System and The Bond Polymer Refine Red Detection System were used for visualization. As chromogen, 3,3diaminobenzidine dyhidrochloride was applied for 10 minutes, and hematoxylin was used as a counterstain for 5 minutes. The entire immunohistochemical procedure was performed with Leica BondMax (Leica Biosystems) autostainer.

The immunoreactivity was estimated as following: keratin 8,18/vimentin: red cytoplasmic/brown cytoplasmic, E-cadherin/ vimentin: brown membranous/red cytoplasmic and E-cadherin/keratin 8,18: brown membranous/red cytoplasmic. Microscopic evaluation and image acquisition was performed with Axiocam 506 color (Zeiss, Jena, Germany). The mesenchymal/epithelial hybrid phenotype cells were quantified at $\times 400$ magnification in three consecutive areas with highest density. For statistical analyses, SPSS 17 software (IBM Analytics, Armonk, NY, USA) was used; differences with $p=0.05$ were considered statistically significant.

\section{Results}

The microscopic evaluation of hematoxylin and eosin-stained liver metastases of digestive origin revealed three histological growth patterns, as following: desmoplastic (28\%), pushing (32\%) and replacement (40\%). All of the liver metastases of pancreatic origin included in the study had a replacement growth pattern, while those with gastric origin had the pushing growth pattern. The colorectal liver metastases exhibited all three histological growth patterns.
Most of the liver metastases described above had G2 tumor grade (48\%), followed by G3 (44\%) and G1 (8\%).

Double immunostaining for E-cadherin/vimentin and keratin 8 or 18/vimentin revealed the following cell types for the desmoplastic (seven cases), pushing (five cases) and replacement histological growth pattern of CRCLM (three cases): epithelial phenotype: E-cadherin ${ }^{+} /$vimentin $^{-}$, keratin $8,18^{+} /$vimentin $^{-}$metastatic cells; mesenchymal phenotype: E-cadherin ${ }^{-} /$vimentin $^{+}$, keratin $8,18^{-} /$vimentin $^{+}$; mesenchymal/ epithelial or non-differentiated hybrid phenotype: E-cadherin ${ }^{+} /$vimentin $^{+}$, keratin $8,18^{+} /$vimentin $^{+}$; and non-hybrid phenotype: E-cadherin ${ }^{-} /$vimentin $^{-}$, keratin $8,18^{-} /$vimentin $^{-}$ (Figure 1a). A heterogeneous expression of E-cadherin, keratin 8,18 with values ranged between 1 to 3 for epithelial phenotype metastatic cells was present. The mesenchymal/epithelial hybrid non-differentiated phenotype cells were noted inside of the areas with decreased E-cadherin, keratin 8,18 expression. All liver metastases presented hybrid phenotype cells. The distribution of hybrid non-differentiated phenotype cells was isolated or in clusters for desmoplastic (Figure 1b and c) and replacement types, and isolated for the pushing type (Figure 1d).

The same heterogeneous patterns of E-cadherin and keratin 8,18 expression were noted in the primary tumors of desmoplastic, pushing and replacement types of colorectal cancer liver metastases. A tendency for non-differentiated hybrid type tumor cells to localize in the basal part of the glands (desmoplastic type) and in the basal (Figure 1e), intermediate and luminal part of the glands (replacement type) was noted. In the primary tumor of replacement model, the luminal hybrid non-differentiated cells (E-cadherin ${ }^{+} /$vimentin $^{+}$; keratin $8,18^{+} /$vimentin $^{+}$) had cellular shape changes, such as hybrid ameboid/mesenchymal morphology (Figure 1f). One desmoplastic type corresponding primary tumor was characterized by the absence of hybrid non-differentiated tumor cells.

In the replacement and pushing histological growth pattern of liver metastases and corresponding primary tumors with pancreatic (seven cases) and gastric (three cases) origin, the heterogeneous profile of E-cadherin/vimentin, keratin 8,18 immunoexpression was maintained. The distribution of hybrid phenotype cells was isolated for pushing type liver metastases growth pattern (gastric; Figure 1g) and isolated or clusters for replacement types (pancreatic origin) types.

The relation between the arithmetic averages of hybrid non-differentiated cells the distribution pattern in primary tumors and the histological growth pattern of liver metastases are summarized in the Table I.

The double immunostaining of E-cadherin and keratin $8 / 18$ revealed the expression of keratin $8 / 18$ to be predominant in hepatic-metastatic cells of colorectal, pancreatic and gastric cancer. The distribution of coexpressing E-cadherin ${ }^{+} /$keratin $8,18^{+}$cells (hybrid differentiated phenotype) was isolated for pushing type 

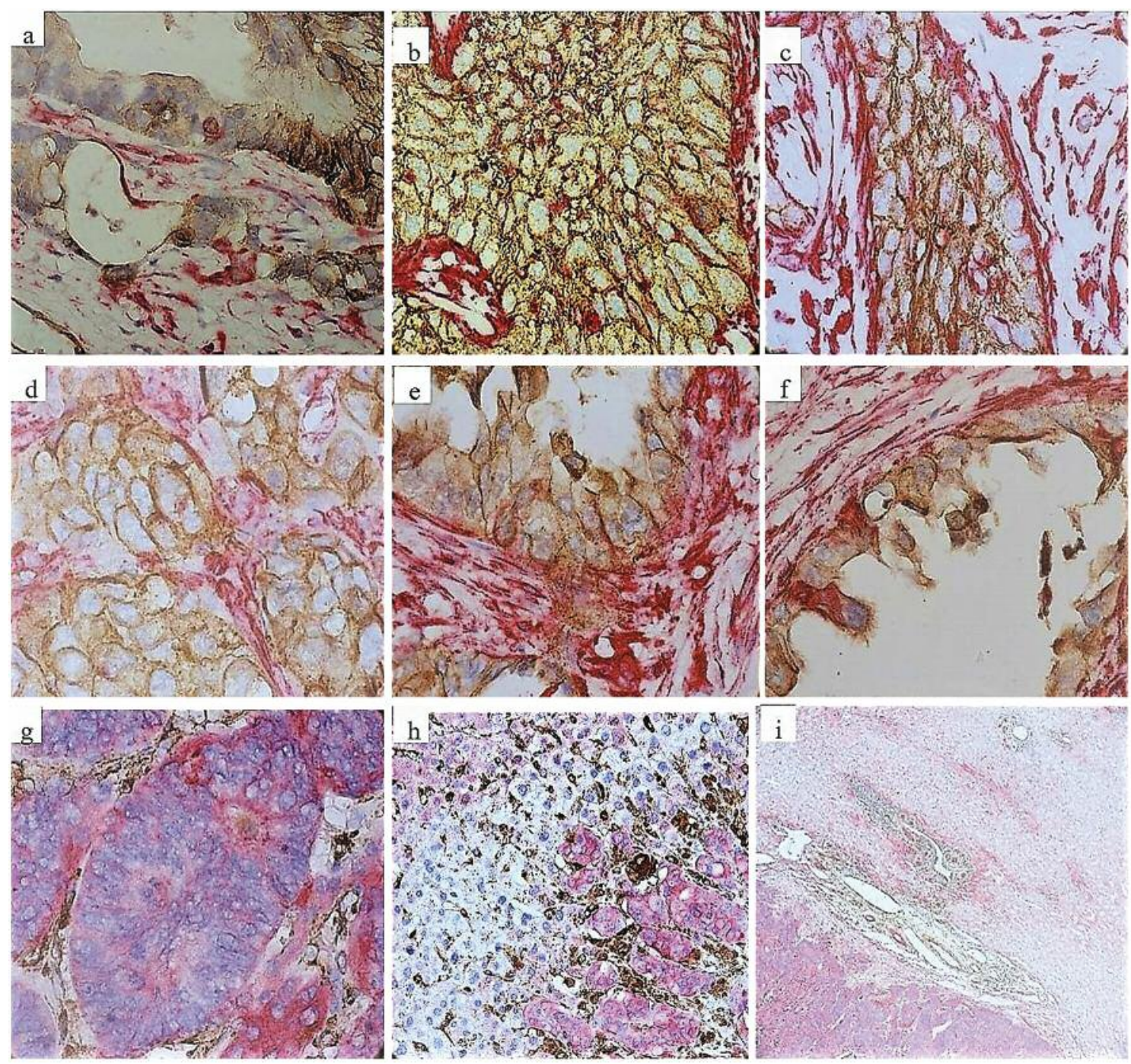

Figure 1. Different patterns of epithelial to mesenchymal transition in liver metastases of various origins. a: Non-hybrid phenotype cells, E-cadherin ${ }^{-} /$vimentin $^{-}$, in the replacement growth pattern of colorectal liver metastases (CRCLM) E-cadherin/vimentin double immunostaining, $\times$ 1,000 magnification. $b$ : Isolated cell with hybrid phenotype in desmoplastic type of CRCLM, E-cadherin/vimentin double immunostaining, $\times 400$ magnification. c: Clusters of hybrid cells, desmoplastic type of CRCLM, E-cadherin/vimentin double immunostaining, x400 magnification. d: Isolated hybrid phenotype cells in the pushing type of CRCLM, E-cadherin/vimentin double immunostaining, x400 magnification.e: Isolated cell basal hybrid phenotype in the replacement type CRCLM, E-cadherin/vimentin double immunostaining, $\times 400$ magnification. f: The amoeboid/mesenchymal morphology of hybrid phenotype cell in the replacement type of CRCLM, E-cadherin/vimentin double immunostaining, $\times 1,000$ magnification. $g$ : Isolated hybrid phenotype cell in the pushing type CRCLM, keratin 8,18/vimentin double immunostaining, $\times 1,000$ magnification. h: Keratin 8,18 expression in metastatic epithelial phenotype cells and hepatocytes in the replacement type of a pancreatic liver metastasis keratin 8,18/vimentin double immunostaining, $\times 400$. i: Keratin 8/18 immunostaining with intensity value of 3 in hepatocytes close to the portal spaces, keratin 8,18/vimentin double immunostaining, $\times 200$ magnification.

(colorectal and gastric) and isolated or clustered cells for replacement and desmoplastic growth pattern of liver metastases (colorectal and pancreatic) types.

Regarding the E-cadherin/vimentin and keratin 8,18/vimentin immunoexpression at the border between metastases and the adjacent liver, all of the cases had higher intensity of E-cadherin and keratin expression in the metastatic epithelial phenotype cell compare with hepatocytes (Figure 1h), with a reduced intensity in the hepatocytes close to metastases, but with increased intensity at a distance from them. In cases with tumor invasion of the portal space, the hepatocytes in close vicinity to the portal space had high intensity of keratin 8/18 and E-cadherin immunoexpression, independent of histological growth pattern of liver metastases (Figure 1i). 
TabIe I. The relation between the arithmetic average of hybrid non-differentiated cells, histological growth pattern of liver metastases and the distribution pattern in primary tumors and liver metastases.

\begin{tabular}{|c|c|c|c|c|c|c|}
\hline \multirow{2}{*}{$\begin{array}{l}\text { Histological growth } \\
\text { pattern of liver metastases/ } \\
\text { primary tumor } \\
\text { CRCLM, desmoplastic type }\end{array}$} & \multicolumn{2}{|c|}{$\begin{array}{l}\text { Hybrid non-differentiated } \\
\text { cells in liver metastases } \\
\left(\text { E-cadherin }{ }^{+} / \text {vimentin }^{+/}\right. \\
\text {primary tumor) }\end{array}$} & \multicolumn{2}{|c|}{$\begin{array}{l}\text { Hybrid non-differentiated } \\
\text { cells (keratin } \\
8,18^{+} / \text {vimentin }^{+/} \\
\text {primary tumor) }\end{array}$} & \multicolumn{2}{|c|}{$\begin{array}{l}\text { The distribution pattern of hybrid } \\
\text { non-differentiated cells in liver } \\
\text { metastases and primary tumors }\end{array}$} \\
\hline & 3.14 & 2 & 3.71 & 2.85 & Isolated or cluster & $\begin{array}{l}\text { Isolated or clusters, } \\
\text { predominantely in the basal } \\
\text { region of the glands }\end{array}$ \\
\hline CRCLM, pushing type & 2.6 & 2.8 & 1.2 & 1.6 & Isolated & Isolated \\
\hline CRCLM, replacement type & 4.33 & 4.66 & 3.33 & 6.66 & Isolated or clusters & $\begin{array}{l}\text { Isolated or clusters; basal, } \\
\text { intermediate and luminal } \\
\text { part of the glands; hybrid, } \\
\text { ameboid/mesenchymal changes }\end{array}$ \\
\hline PLM, replacement type & 2 & 4.85 & 3 & 5.42 & Isolated or clusters & Isolated or clusters \\
\hline GLM, pushing type & 4 & 7 & 4 & 5 & Isolated & Isolated \\
\hline
\end{tabular}

CRCLM: Colorectal liver metastases, PLM: pancreatic liver metastases, GLM: gastric liver metastases.

For the primary colorectal cancer tumors, significant correlation was found between the presence of nondifferentiated hybrid phenotype cells (E-cadherin ${ }^{+} /$vimentin $^{+}$; keratin $8,18^{+} /$vimentin $^{+}$) and the histological growth pattern $(p=0.003 ; p=0.025)$. Significant correlation was noted between the number of non-differentiated hybrid phenotype cells (keratin $8,18^{+} /$vimentin $^{+}$) and differentiated hybrid phenotype (keratin $8,18^{+} /$E-cadherin $^{+}$) in primary tumors, and those in liver metastases: $p=0.009$ and $p=0.044$, respectively. A significant correlation was noted between the number of differentiated hybrid phenotype cells (E-cadherin ${ }^{+} /$keratin $\left.8,18^{+}\right)$and of non-differentiated hybrid phenotype cells ${\text { (keratin } 8,18^{+} / \text {vimentin }^{+}, p=0.05 ; \text { E-cadherin }}^{+} /$vimentin $^{+}$, $p=0.032$ ) in liver metastases. A similar association was present in primary tumor $(p=0.050)$.

For pancreatic cancer, a significant correlation was found between the number of differentiated hybrid phenotype cells and differentiation grade $G$ in primary tumor and corresponding liver metastases $(p=0.05)$.

A significant correlation between the number of nondifferentiated hybrid phenotype cells (E-cadherin ${ }^{+} /$ vimentin ${ }^{+}$) and the histological growth pattern characterized gastric cancer $(p=0.005)$.

\section{Discussion}

In normal situations, E-cadherin, a calcium-dependent celladhesion molecule is necessary for epithelial histogenesis, tissue stabilization, differentiation and induction of EMT during embryogenesis $(21,22)$. In pathological situations, abnormal expression of E-cadherin and $\beta$-catenin favor the mesenchyme phenotype cell (23).
Keratin 8,18 expression in normal conditions was found in simple epithelium (liver, pancreas, kidney), mixed epithelium (breast, lung) and is involved in embryogenesis $(24,25)$. In pathological situations, its increased expression was noted in adenocarcinomas and squamous cell carcinoma with different localization $(26,27)$. The mains roles of keratin $8 / 18$ were: modulation of protein localization, protein targeting and apoptosis (28).

Data from the literature show that MET is a part of the metastatic process, in which the tumor cells regain epithelial properties at their secondary site $(29,30)$. It was noted that metastatic lesions had the same features of epithelial immunoexpression markers as primary tumors $(31,32)$. This pattern was found in our study. The hybrid differentiated cell type (E-cadherin ${ }^{+} /$keratin $8,18^{+}$) was found in metastatic cells. Significant correlation between the presence of co-expressing E-cadherin/keratin 8/18 cells in primary tumor and colorectal cancer liver metastases was found also. These observations may support the idea that a constant number of hybrid differentiated cells from primary tumors migrate to the secondary organ and maintain the same phenotype there. The existence of significant correlation between the non-differentiated hybrid phenotype cells in primary tumor and differentiated hybrid phenotype cells in colorectal cancer liver metastases argues for EMT/MET and MET/EMT plasticity of some tumor cells. This hypothesis was sustained by a significant correlation between non-differentiated and differentiated hybrid phenotype cells in both primary tumor and liver metastases of colorectal carcinoma.

Strauss et al. showed that some cells with hybrid epithelial/mesenchymal phenotype in primary ovarian 
cultures and tumors in situ can be multipotent, express markers of other lineages, and drive tumor growth in vivo by giving rise to another epithelial/mesothelial subset as well as completely differentiated epithelial cells (33). Partial loss of E-cadherin expression was associated with carcinoma progression and unfavorable prognosis. In the final stages of numerous carcinomas, E-cadherin expression appears to be heterogeneous, with E-cadherin ${ }^{-}$tumor cells interposed between E-cadherin ${ }^{+}$tumor cell areas, suggesting that certain carcinoma cells have EMT properties (34). The same heterogeneous phenotype cells, with E-cadherin ${ }^{+} /$vimentin $^{-}$, E-cadherin ${ }^{+} /$keratin $8,18^{+}$, keratin $8,18^{+} /$vimentin $^{-}$cells between cells without immunoexpression of epithelial markers were noted in the liver metastases and corresponding primary tumors analyzed in the present study. Over $75 \%$ of circulating tumor cells in women with metastatic breast cancer were found to co-express epithelial marker, mesenchymal marker $N$-cadherin, and stem cell markers (35).

It was shown for the patients with replacement growth pattern of CRCLM the hazard of death was 2-2.5 times higher than for patients with pushing growth or mixed growth pattern, and nearly 4-times higher than for patients with desmoplastic growth pattern. The negative prognostic effect of the replacement growth pattern was even more pronounced after adjusting for tumor size (20). These findings correspond to the highest values of differentiated and non-differentiated hybrid cell types for the replacement histological growth pattern. A significant correlation was found between the histological growth pattern and frequency of cells with nondifferentiated phenotype in colorectal and gastric cancer.

EMT was also observed in other cancer types and also in their corresponding metastases (36). Thus, EMT has already become a target for several different therapeutic agents tested in experimental or preclinical studies $(37,38)$.

\section{Conclusion}

This study suggests the existence of significant correlation between the presence of non-differentiated hybrid phenotype cells in primary tumor and differentiated hybrid phenotype cells in colorectal cancer liver metastases. These support the hypothesis of EMT/MET and MET/EMT plasticity of some tumor cells in liver metastases. The existence of significant correlation between the cells with non-differentiated hybrid phenotype and the histological growth pattern in colorectal and gastric cancer indicates the potential for an MET-targeting strategy, in conjunction with conventional chemotherapy, for treatment of liver metastases of digestive origin.

\section{Conflicts of Interest}

None declared.

\section{Acknowledgements}

The Authors are grateful to Patricia Berzava and Ciprian Onica for their excellent technical support.

\section{References}

1 Jolly MK, Boareto M, Huang B, Jia D, Lu M, Ben-Jacob E, Onuchic JN and Levine $\mathrm{H}$ : Implications of the hybrid epithelial/mesenchymal phenotype in metastasis. Front Oncol 5(155): 1-19, 2015.

2 Thiery JP, Acloque H, Huang RYJ and Nieto MA: Epithelialmesenchymal transitions in development and disease. Cell 139(5): 871-890, 2009.

3 Kalluri $\mathrm{R}$ and Weinberg RA: The basics of epithelialmesenchymal transition. J Clin Invest 119: 1420-1428, 2009.

4 Zeisberg $M$ and Neilson EG: Biomarkers for epithelialmesenchymal transitions. J Clin Invest 119: 1429-1437, 2009.

5 Brabletz T, Jung A, Reu S, Porzner M, Hlubek F, KunzSchughart LA, Knuechel R, and Kirchner T: Variable betacatenin expression in colorectal cancers indicates tumor progression driven by the tumor environment. Proc Natl Acad Sci USA 98: 10356-10361, 2001.

6 Li A, Omura N, Hong SM, Vincent A, Walter K, Griffith M, Borges $\mathrm{M}$ and Goggins $\mathrm{M}$ : Pancreatic cancers epigenetically silence SIP1 and hypomethylate and overexpress miR-200a/200b in association with elevated circulating miR-200a and miR-200b levels. Cancer Res 70: 5226-5237, 2010.

7 Winter JM, Ting AH, Vilardell F, Gallmeier E, Baylin SB, Hruban RH, Kern SE and Iacobuzio-Donahue CA: Absence of E-cadherin expression distinguishes noncohesive from cohesive pancreatic cancer. Clin Cancer Res 14: 412-418, 2008.

8 Rosivatz E, Becker I, Specht K, Fricke E, Luber B, Busch R, Höfler $\mathrm{H}$ and Becker KF: Differential expression of the epithelial-mesenchymal transition regulators snail, SIP1, and twist in gastric cancer. Am J Pathol 161: 1881-1891, 2002.

9 Tarin D, Thompson EW and Newgreen DF: The fallacy of epithelial-mesenchymal transition in neoplasia. Cancer Res 65(14): 5996-6000, 2005.

10 Thompson EW, Newgreen DF and Tarin D: Carcinoma invasion and metastasis: A role for epithelial-mesenchymal transition? Cancer Res 65(14): 5991-5995, 2005.

11 Gao D and Mittal V: Tumor microenvironment regulates epithelial-mesenchymal transitions in metastasis. Expert Rev Anticancer Ther 12(7): 857-859, 2012.

12 Yates C: Prostate tumor cell plasticity: a consequence of the microenvironment. Adv Exp Med Biol 720: 81-90, 2011.

13 Hur K, Toiyama Y, Takahashi M, Balaguer F, Nagasaka T, Koike J, Hemmi H, Koi M, Boland CR and Goel A: MicroRNA-200c modulates epithelial-to-mesenchymal transition (EMT) in human colorectal cancer metastasis. Gut 62: 1315-1326, 2013.

14 Gurzu S, Turdean S, Kovecsi A, Contac AO and Jung I: Epithelial-mesenchymal, mesenchymal-epithelial, and endothelial-mesenchymal transitions in malignant tumors: An update. World J Clin Cases 3(5): 393-404, 2015.

15 Chou CK, Fan CC, Lin PS, Liao PY, Tung JC, Hsieh CH, Hung $\mathrm{MC}$, Chen $\mathrm{CH}$, and Chang WC: Sciellin mediates mesenchymalto-epithelial transition in colorectal cancer hepatic metastasis. Oncotarget 7(18): 25742-25754, 2016. 
16 Aiello NM, Bajor DL, Norgard RJ, Sahmoud A, Bhagwat N Pham MN, Cornish TC, Donahue CAI, Vonderheide RH and Stanger BZ: Metastatic progression is associated with dynamic changes in the local microenvironment. Nat Commun 7: 12819, 2016.

17 Vermeulen PB, Colpaert C, Salgado R, Royers R, Hellemans H and Van Den Heuvel E: Liver metastases from colorectal adenocarcinomas grow in three patterns with different angiogenesis and desmoplasia. J Pathol 195: 336-342, 2001.

18 Eefsen R, Van den Eynden G, Brodt P, Burnier J, Høyer-Hansen $\mathrm{G}$ and Laerum $\mathrm{O}$ : Multiple liver metastases from the same patient exhibit a uniform growth pattern. ASCO GI-Symposium; 19-21; San Francisco, CA. Abstract no 8770, 2012.

19 Simoneau E, Vermeulen P, Van den Eynden G, Reynolds A, Petrillo S, Valenti D and Metrakos P: The histological growth patterns of colorectal cancer liver metastasis are associated with disease progression post portal vein embolization. HPB J 19(1): S1-S192, 2017.

20 Nielsen K, Rolff HC, Eefsen RL and Vainer B: The morphological growth patterns of colorectal liver metastases are prognostic for overall survival. Mod Pathol 27: 1641-1648, 2014.

21 Marrs JA and Nelson WJ: Cadherin cell adhesion molecules in differentiation and embryogenesis. Int Rev Cytol 165: 159-205, 1996.

22 Brown JD and Moon RT: Wnt signaling: why is everything so negative? Curr Opin Cell Biol 10: 182-187, 1998.

23 Rubinfeld B, Rubins P, El-Gamil M, Albert I, Porfiri E and Polakis P: Stabilization of beta-catenin by genetic defects in melanoma cell lines. Science 275: 1790-1792, 1997.

$24 \mathrm{Ku}$ NO and Omary MB: A disease- and phosphorylation-related nonmechanical function for keratin 8. J Cell Biol 174: 115-125, 2006.

25 Larue L and Bellacosa A: Epithelial-mesenchymal transition in development and cancer: role of phosphatidylinositol 3' kinase/AKT pathways. Oncogene 24: 7443-7454, 2005.

26 Fortier AM, Asselin E and Cadrin M: Functional specificity of Akt isoforms in cancer progression. Biomol Concepts 1-2: 1-11, 2011.

27 Zhang S and Yu D: PI(3)king apart PTEN's role in cancer. Clin Cancer Res 16: 4325-4330, 2010.

28 Galarneau L, Loranger A, Gilbert S and Marceau N: Keratins modulate hepatic cell adhesion, size and $\mathrm{G}_{1} / \mathrm{S}$ transition. Exp. Cell Res 313: 179-194, 2007.
29 Hugo H, M. Ackland L and Blick T: Epithelial-mesenchymal and mesenchymal-epithelial transitions in carcinoma progression. J Cell Physiol 213(2): 374-383, 2007.

30 Yao D, Dai C and Peng S: Mechanism of the mesenchymalepithelial transition and its relationship with metastatic tumor formation. Mol Cancer Res 9(12): 1608-1620, 2011.

31 Chao YL, Shepard CR and Wells A: Breast carcinoma cells reexpress E-cadherin during mesenchymal to epithelial reverting transition. Mol Cancer 9: 179, 2010.

32 Krawczyk N, Meier-Stiegen F, Banys M, Neubauer H, Ruckhaeberle E and Fehm T: Expression of stem cell and epithelial-mesenchymal transition markers in circulating tumor cells of breast cancer patients. BioMed Res Int 415721: 1-11, 2014.

33 Strauss R, Li Z-Y, Liu Y, Beyer I, Persson J and Sova P: Analysis of epithelial and mesenchymal markers in ovarian cancer reveals phenotypic heterogeneity and plasticity. PLoS One 6: e16186, 2011.

34 Imai T, Horiuchi A and Shiozawa T: Elevated expression of Ecadherin and $\alpha_{-}, \beta-$, and $\gamma$-catenins in metastatic lesions compared with primary epithelial ovarian carcinomas. Hum Pathol 35(12): 1469-1476, 2004.

35 Armstrong AJ, Marengo MS, Oltean S, Kemeny G, Bitting RL and Turnbull JD: Circulating tumor cells from patients with advanced prostate and breast cancer display both epithelial and mesenchymal markers. Mol Cancer Res 9: 997-1007, 2011.

36 Tsoukalas N, Aravantinou-Fatorou E, Tolia M, Giaginis C, Galanopoulos M, Kiakou M, Kostakis ID, Dana E, Vamvakaris I, Korogiannos A, Tsiambas E, Salemis N, Kyrgias G, Karameris A and Theocharis S: Epithelial-mesenchymal transition in non small-cell lung cancer. Anticancer Res 37(4): 1773-1778, 2017.

37 Tungsukruthai S, Sritularak $B$ and Chanvorachote P: Cycloartobiloxanthone inhibits migration and invasion of lung cancer cells. Anticancer Res 37(11): 6311-6319, 2017.

38 Ryu SH, Heo SH, Park EY, Choi KC, Ryu JW, Lee SH, and Lee SW: Selumetinib inhibits melanoma metastasis to mouse liver via suppression of EMT-targeted genes. Anticancer Res 37(2): 607-614, 2017

Received November 18, 2017

Revised January 1, 2018

Accepted January 4, 2018 